\title{
¿Qué esperan obtener los jóvenes chilenos de las marcas que siguen en Redes Sociales?
}

\section{Chilean young people: What do they expect to get when they follow a brand on social networks?}

\author{
Guillermo Bustamante Pavez \\ Profesor asociado Facultad de Comunicaciones \\ Director de la Escuela de Comunicación Digital y Multimedia \\ Investigador Responsable Núcleo Académico Media Garage \\ (Universidad del Pacífico)
}

Fecha de recepción: 2 de mayo de 2017

Fecha de revisión: 19 de junio de 2017

Fecha de publicación: 1 de julio de 2017

Para citar este artículo: Bustamante Pavez, G. (2017): ¿Qué esperan obtener los jóvenes chilenos de las marcas que siguen en Redes Sociales?, Icono 14, volumen 15 (2), pp. 115137. doi: $10.7195 /$ ri14.v15i2.1085 


\section{MONOGRÁFICO}

\section{Resumen}

Con el objetivo de comprender las motivaciones de los jóvenes universitarios chilenos al momento de seguir a una marca en redes sociales y a través de ello, revelar el valor percibido por ellos de las marcas en canales digitales es que se realizó un estudio exploratorio cuantitativo a través de la aplicación de una encuesta autoadministrada en línea con una muestra de 1.117 jóvenes chilenos matriculados en una Institución de Educación Superior durante 2016.

Este estudio, reveló las motivaciones y el valor percibido que tienen de las marcas en canales digitales. En este punto, lo más valorado por los encuestados, respecto de lo que realizan las marcas en redes sociales, es el contenido que comparten (64\%) que las marcas realicen promociones, concursos o den regalos (21\%) las respuestas y soluciones a problemas (8\%) y la utilidad de la información que publican (7\%).

\section{Palabras clave}

Jóvenes y redes sociales - Marcas y redes sociales - Comunicación digital

\section{Abstract}

With the objective of understanding the motivations of young Chilean university students to follow a brand in social networks and through this, revealing the perceived value of brands in digital channels is that a quantitative exploratory study was carried out through the application of a self-administered online survey. The sample was 1,117 young Chilean university students enrolled in a Higher Education Institution during 2016.

This study revealed the motivations and perceived value of brands in digital channels. At this point, what is most valued by respondents, with respect to what brands do in social networks, is the content (64\%) that brands make promotions, contests or give gifts (21\%) the answers and solutions to Problems (8\%) and the usefulness of the information they publish (7\%).

\section{Key Words}

Young people and social networks - Brands and social networks - Digital comunication 


\section{Introducción}

Desde la irrupción de las redes sociales como canal de comunicación para las marcas la indagación sobre esta temática ha incorporado nuevos espacios. Es así como la investigación de Martín, E. y Sánchez-Martín, L. (2011) explora sobre la nueva relación entre marca-producto-consumidor en estos entornos, mientras que lo planteado por Soengas, X., Vivar, H. y Abuín, N (2015) se centra en los formatos idóneos para llegar a los usuarios y Castelló, A. (2010) aborda la capacidad de creación y participación que adquiere el usuario en los espacios de la web 2.0.

Todos estos estudios coinciden en que las redes sociales han provocado un empoderamiento de los usuarios, que han pasado de ser meros consumidores de información y receptores de contenidos a convertirse en gestores y productores de los mismos. Opinando, comprometiéndose y dominando -en la gran mayoría de las ocasiones- el lenguaje del marketing y la publicidad (Castelló, A. 2010)

Las investigaciones realizadas coinciden en que los jóvenes son el principal usuario de los nuevos medios de comunicación derivados del desarrollo de las nuevas tecnologías y, por consiguiente, uno de los objetivos más importantes de las estrategias publicitarias de las empresas en esos medios (Muela, C. y Baladrón, A. (2010); Bernal, C y Angulo, F. (2013))Lamentablemente no hay estudios que exploren sobre la relación con las marcas y los que existen solo centran la mirada en las motivaciones de los usuarios y la relación de éstos con sus pares equivalentes.

El objetivo general de esta investigación es explicar el valor percibido por los estudiantes de Educación Superior Chilenos matriculados durante 2016 que siguen a marcas en Redes Sociales.

Para lograr este objetivo a) se identificó el valor percibido por los estudiantes de Educación Superior Chilenos matriculados durante 2016 que siguen a marcas en Redes Sociales b) se categorizó el valor percibido por los estudiantes de Educación Superior Chilenos matriculados durante 2016 que siguen a marcas en Redes Sociales de acuerdo que la ecuación planteada por Bonal S. y Cuadrado, J. (2004) complementada con la Tipología de Holbrook, M.B. (1999) c) se comparó el valor 


\section{MONOGRÁFICO}

percibido que le dan los estudiantes de Educación Superior Chilenos matriculados durante 2016 a las marcas de diferentes industrias que siguen en Redes Sociales y finalmente e) se determinó las categorías cuyas marcas presentan mejor valor percibido en Redes Sociales por los estudiantes de Educación Superior Chilenos matriculados durante 2016.

\section{Marcas y Redes Sociales}

Según Igarza (2008) “los medios sociales son plataformas que permiten crear o mantener la comunicación en línea entre los miembros de una comunidad" lo que es tomado como base por Rosales (2010) cuando describe los tipos de medios a utilizar en una estrategia digital. Sobre ellos y el rol que juegan para las marcas señala que "no se trata de extrapolar los típicos formatos publicitarios que teníamos hasta ahora, sino de contextualizar lo publicidad. No se trata de anunciarse, sino de relacionarse".

Por otra parte, Bernal, C. y Angulo, F. (2013) señalan respecto de las redes sociales que éstas son "un servicio de Internet que permite a cualquier cibernauta construirse un perfil -público o semi público- dentro de un sistema gestionado por un tercero; compartir relaciones- experiencias o contenido, con una lista de otros usuarios".

En este espacio Martín, E. y Sánchez-Martín, L. (2011) señalan que “las redes sociales han configurado una nueva relación entre marca-producto-consumidor. Por una parte, los productos que eran el elemento más relevante de la publicidad han sido sustituidos por la marca en entornos digitales"

Lo anterior, porque según Soengas, X., Vivar, H. y Abuín, N (2015) “los formatos tradicionales ya no llegan a los consumidores que quieren participar del mensaje publicitario lo que obliga a los creativos a desarrollar nuevas fórmulas"

Por ello es que de acuerdo a Martín, E. y Sánchez-Martín, L. (2011) las marcas "utilizan estos perfiles para entablar una comunicación personalizada entre ellos y sus clientes. La tecnología actual y concretamente las redes sociales permiten co- 
nocer más que nunca a las audiencias facilitando así el ideal de la personalización de los contenidos y de la publicidad one to one".

Es que, de acuerdo a lo señalado por Castelló, A. (2010) “los social media han traído consigo nuevas formas de consumo del medio derivadas de la mayor capacidad de creación y participación que adquiere el usuario en los espacios de la web $2.0^{\prime \prime}$

A lo anterior, la misma autora agrega que "existe una serie de factores que están transformando el modelo de comunicación empresarial: el incremento de clientes más inteligentes y exigentes con la calidad, la avalancha de mensajes comerciales, los nuevos líderes de opinión como los bloggers, la pérdida de credibilidad de los medios tradicionales y las comunidades derivadas de las nuevas tecnologías".

En este espacio, es necesario verificar de qué forma perciben e interpretan los usuarios a las marcas y cómo éstas comparten contenidos. Es acá, donde desde el análisis semiótico se puede dar más valor a los mensajes emitidos por las marcas y que generan más recordación para los usuarios.

\section{Jóvenes y Motivaciones para el uso de Redes Sociales}

Tal y como explican Soengas, X., Vivar, H. y Abuín, N (2015) “las redes sociales han provocado un empoderamiento de los usuarios, que han pasado de ser meros consumidores de información y receptores de contenidos a convertirse en gestores y productores de los mismos. En la actualidad, cualquier usuario con conocimientos básicos de ofimática puede producir y difundir contenidos globalmente debido a la gran capacidad viral de los medios sociales".

De acuerdo a Castelló, A. (2010) "el protagonista de este escenario digital e interactivo es alguien que opina, se compromete y dominan -en la gran mayoría de las ocasiones- el lenguaje del marketing y la publicidad; un consumidor experto que codifica las intenciones de las campañas publicitarias y las estrategias de marca". 


\section{MONOGRÁFICO}

Soengas, X., Vivar, H. y Abuín, N (2015) puntualizan que "estos consumidores son activos productores de información sobre las marcas que consumen y uno de los pasos que cierra el ciclo del proceso de compra es la publicación de su opinión en los medios sociales tanto que si ésta es positiva como negativa".

Lo relevante es lo que plantean Muela, C. y Baladrón, A. (2010) quienes revelan que "la juventud es el principal usuario de los nuevos medios de comunicación derivados del desarrollo de las nuevas tecnologías y por consiguiente, uno de los objetivos más importantes de las estrategias publicitarias de las empresas en esos medios".

Esto, devela la necesidad de entender cómo los jóvenes ven a las marcas que realizan comunicación en redes sociales, porque según Bernal, C y Angulo, F. (2013) "diversos estudios sobre jóvenes y redes sociales han demostrado en general el alto consumo de estos servicios, generalizando su uso en casi todo tipo de ubicaciones y valorando su importancia en la construcción de las relaciones entre la juventud."

Es en este espacio, que a todos los nacidos desde 1980 se les conoce como "Generación Y" o "Millenials". Este grupo ha crecido en una época caracterizada por los avances tecnológicos, como las redes sociales las que forman parte de su vida: los reconocen, los utilizan y los desarrollan. (Barford \& Hester, 2011).

Según, Carvallo (2014) corresponden al grupo etáreo nacido entre 1980 y 1995. En los últimos años, ellos han entrado al mundo laboral, y están demostrando que tienen una visión del mundo y del futuro muy diferente a la que tenían sus padres, los que en su mayoría pertenecen a la llamada "Generación X" o a la anterior, "Generación del Baby Boom".

Además, son independientes, individualistas y firmes creyentes de que el éxito es un camino y no una meta. Además, los jóvenes que pertenecen a esta generación buscan permanentemente un balance adecuado entre trabajo y vida social/ familiar. (Connell, McMinn, \& Bell, 2012)Es importante destacar, que la generación Y impulsa la diversidad, no por aceptación de la diferencia, sino por eliminación directa de la etiqueta (Mason, 2013). 
De acuerdo a la, "Encuesta Telefónica Global Millennial: Foco en Chile" desarrollado por Telefónica en 2013, “los Millenial Chilenos están muy empoderados por la tecnología, pero también valoran altamente los medios tradicionales"

A modo de resumen, el estudio anterior concluye que "son usuarios ávidos de tecnología, cerca de los tres cuartos de los millennial chilenos usan smartphones (73\%) y permanecen diariamente en promedio siete horas online, cifra superior al promedio global de seis horas"

Otros datos relevantes que se encuentran en el estudio elaborado por Telefónica y que sirven de contexto para lo trabajado en esta investigación son los siguientes:

- Un tercio (33\%) de ellos consideran Internet como el medio de cobertura informativa más idóneo y creíble (37\% a nivel mundial) y más de la mitad de ellos, el $62 \%$ declara a Internet como su principal centro de entretenimiento (53\% a nivel global)

- $35 \%$ de los millennial chilenos aseguran que van a la par de la última tecnología existente en el Mercado (30\% nivel global)

- Cerca de la mitad de la muestra local, $44 \%$, describe su conocimiento personal de la tecnología como excelente ( $30 \%$ a nivel mundial)

Un factor importante que el empoderamiento tecnológico de este grupo en Chile que es mucho más alto que el de sus pares a nivel mundial:

- $91 \%$ creen que la tecnología permite disminuir o sobrellevar las barreras del lenguaje ( $87 \%$ a nivel mundial)

- $89 \%$ de los encuestados cree que la tecnología facilita el encontrar un trabajo ( $83 \%$ global)

- Más de dos cuartos de la muestra (76\%) cree que la tecnología crea oportunidades para todos (69\% total) 
Los millennial chilenos (54\%) creen que la tecnología produce un efecto balanceador en la sociedad chilena, manifestando que ésta ha disminuido la brecha entre ricos y pobres (38\% global)

Todo lo anterior y considerando, además, las investigaciones realizadas por Bernal, C. y Angulo, F. (2013) sobre las interacciones de los jóvenes en redes sociales (medios sociales) Muela, C. y Baladrón, A. (2011) sobre los espacios formas y retos de realizar publicidad enfocada en jóvenes y de Alvarado, P. (2012) respecto del impacto de las redes sociales (medios sociales) sobre las variables de decisiones de los agentes (usuarios) son una nueva puerta al estudio de un área poco explorada: las valoración que realizan los usuarios de medios sociales respecto de la incorporación de las marcas en este tipo de medios y cuál es la utilidad que los usuarios le dan a la comunicación que realizan las marcas en estos canales.

En ese contexto y desde el punto de vista de las interacciones es que la investigación de Bernal, C. y Angulo, F. (2012) clarifica las motivaciones que tienen los jóvenes para usar las redes sociales señalando que se pueden dividir en tres categorías: motivación social, psicológico-afectiva y la vida cotidiana. Desde estas tres categorías es que se puede abordar la relación que los jóvenes tienen con las marcas que deciden incorporarse en medios sociales.

Algo que es complementado por Muela, C. y Baladrón, A. (2010) quienes explican que "otro aspecto fundamental es la tendencia en esa publicidad para jóvenes a incidir más en los aspectos emocionales que en los racionales, impulsada precisamente por el referido carácter informado de la juventud

Los trabajos actuales se centran en determinar qué es lo que motiva el uso de Redes Sociales, siendo en este contexto, los jóvenes, y según lo señalado por Zheng, R. y Cheok, A. (2011) el grupo etario al que es necesario tener con los datos más actualizados posibles pues son los que presentan un crecimiento más rápido en la adopción de nuevas tecnologías.

Desde esta lógica y según lo que plantean Colás, P., González, T., y de Pablos, J. (2012) "las redes sociales on-line son para la juventud fuente de recursos que 
son utilizados para cubrir necesidades, tanto de índole psicológica como social. Sin embargo, las diferencias entre sexos en estas variables demuestra que tienen un papel compensatorio, ya que son los hombres los que mayormente recurren a ellas para cubrir facetas emocionales y reforzar su autoestima, mientras en el caso de las jóvenes prima una función relacional.

Basado en lo anterior, parece importante destacar el estudio de Notley, T. (2009) que identifica los factores clave que afectan al uso que los jóvenes y adolescentes australianos le dan a las redes sociales. A partir de este estudio, el autor plantea un modelo teórico explicativo de cuatro dimensiones basado en el uso que le dan los jóvenes australianos a las redes sociales y que puede ser extrapolado a la realidad chilena.

El mismo autor, plantea que existen cuatro factores que determinan la relación de los jóvenes con las Redes Sociales: intereses personales, necesidades, relaciones y competencias tecnológicas.

Según el estudio Wave realizado en 2014 por la agencia de publicidad Universal Mccann se ha dado un paso importante desde la motivación y uso de las redes sociales como elementos de diversión al del aprendizaje.

El mismo estudio revela que entre los usos potenciales de las redes sociales se encuentran: ganar respeto, aprender sobre algo nuevo, hacer dinero y buscar opiniones de otras personas.

Lamentablemente el estudio anterior no explora sobre la relación con las marcas y solo centra la mirada en las motivaciones de los usuarios y la relación de éstos con sus pares equivalentes.

A pesar de ello, en este estudio se identifican las principales motivaciones para participar de una red social que de acuerdo a Rogers, M (2014) pueden agruparse de la siguiente manera: 


\section{MONOGRÁFICO}

Relación: El aspecto social de las redes es la motivación principal. El contacto con resto de las personas, sentirse parte de una comunidad, paliar la soledad, constituyen el leit motiv para utilizar redes sociales.

Diversión: Escapar del cotidiano y del consecuente tedio, por medio de la relación social es el segundo punto más fuerte.

Progreso: El contenido permite estar más allá de las limitaciones promedio de cualquier sector.

Reconocimiento: Se comparte contenido para buscar más RT y Likes; se comparte contenido buscando reconocimiento.

Aprendizaje: La valoración del contenido tiene directa relación con el aprendizaje y por tanto está relacionado con el reconocimiento y el progreso.

Respecto de la relación de los usuarios de redes sociales y las marcas según lo señalado por Antonio Traugott, director general de IAB Spain, en el VI Estudio Anual de Redes Sociales (2014) "las redes sociales siguen con su proceso de madurez y consolidación entre consumidores y marcas. Los datos de penetración son muy altos, pero personalmente destaco el hecho de que un $89 \%$ de usuarios sigan a alguna marca; eso quiere decir que las empresas están cada vez más adaptadas a este terreno y están encontrando formas no intrusivas de conectar con la audiencia".

Desde los datos recolectados en el estudio antes mencionado se concluye que “las principales razones por las que los internautas deciden seguir a marcas en redes sociales son mantenerse informados, participar en un concurso creado por la marca, para conocer su funcionamiento o bien porque influye en su proceso de compra".

Algo similar a lo anterior, concluyó el estudio realizado por Cruz, B. y Mendelsohn, J. (2010) de las empresas norteamericanas Chadwick Martin Bailey e iModerate en donde se encuestó a usuarios activos de Facebook de Estados Unidos y que reveló que el 25\% sigue las marcas para conseguir promociones y descuentos, por lo que se demuestra el interés en comprar sus productos o servicios, el $21 \%$ es 
consumidor de esa marca y al 18\% le gusta relacionar su identidad a la de la marca, como las principales razones de uso.

Lehr, A (2015) lideró a un equipo de las agencias de Relaciones Públicas y de Marketing BuzzStream y Fractl que encuestó a 900 usuarios estadounidenses de medios sociales tras lo que concluyeron que lo que se esperan de las marcas es:

- Contenido exclusivo (y por tanto nuevo)

- Relevante y relacionado con la marca y sus productos o servicios

- Un compromiso con los seguidores en cuanto a capacidad de respuesta, evitando las respuestas de tipo

- Una actividad regular

Respecto de las razones que hacen que una persona deje de seguir a una marca, el mismo estudio puntualiza los siguientes motivos

- El 21\% por considerar el contenido repetitivo o aburrido

- El $19 \%$ porque la marca hace spam, lo que se considera si actualiza más de seis veces al día

- El $17 \%$ por aliviar sus timelines

- El $16 \%$ por considerar que la actividad de la marca es ofensiva o molesta

- El $14 \%$ porque el contenido no tiene nada que ver con la marca y sus actividades

- El $8 \%$ porque la marca no responde o es demasiado lenta a la hora de ofrecer feedback

- $\quad$ El 7\% porque deja de ser cliente de la marca. 


\section{Valor y Valor percibido}

Según Hernández, A. (2012) citando Gil et. al., (2006) el concepto de valor es propio de numerosos dominios y concretamente en el ámbito del marketing se aborda desde las perspectivas del precio, estrategia y comportamiento del consumidor.

Debido a ello, la misma autora agrega que "no existe un concepto único y homogéneo sobre valor percibido admitido unánimemente por la literatura, por el contrario, hemos encontrado diversas concepciones en un intento de explicación del constructo siguiendo distintos puntos de vista."

A pesar de ello y según puntualiza la misma autora, el valor percibido "está casi siempre relacionado con diversas variables como la calidad, la lealtad y la satisfacción".

Todo esto, ha provocado dificultades en la investigación sobre el concepto de valor tal como señalan las investigaciones realizadas por Dodds, W., Monroe, K. y Grewal, D. (1991); Babin, B.J., Darden, W.R. y Griffin, M. (1994); Anderson, J.C. (1995); Woodruff B. R. y Gardial F. S. (1996); Gabbott, M. y Hogg, G. (1998) Bigné, J.E., Moliner, M.A. y Callarisa, L.J. (2000); Sweeney, J. y Soutar, G. (2001)

A lo anterior, es necesario agregar lo que el valor, según Gallarzar, M. y Gil, I. (2005), es una noción difícil de delimitar conceptualmente respecto de otras nociones afines y que como bien señala Woodruff B. R. y Gardial F. S. (1996) ,la mayoría de las propuestas de definición de valor se apoyan en conceptos como la calidad y la satisfacción, cuyo significado en la literatura resulta igualmente esquivo y poco claro. Se podría decir que son conceptos híbridos que además resultan ser todos de capital importancia para la investigación de marketing.

Considerando todo lo planteado es que se entenderá valor y valor percibido de las siguientes maneras: 
Valor: Es una experiencia interactiva, relativa y preferencial Holbrook, M.B. (1999), un vínculo emocional que se establece entre un cliente y productor (Butz, H.E. y Goldstein, L.B. (1996) ) que se expresa en las ventajas que se perciben a cambio de las cargas que se soportan (Berry, L.D. y Yadav, M.S. (1997))

Valor Percibido: Es un equilibrio entre la calidad o beneficios percibidos en un producto y el sacrificio percibido por el pago de un precio (Monroe K. B. (1992)) o la expresión del consumidor de los beneficios del producto (Nilson, T.H. (1992))

\section{Tipologías y escalas de valor}

Según Gallarzar, M. y Gil, I. (2005) uno de los escasos consensos en torno al valor es la necesidad de abordarlo de forma integrada y entendiendo que no se puede comprender qué es valor sin entender la diferencia de un tipo de valor respecto de otro.

Uno de los trabajos más completos sobre las tipologías del valor (ver figura $\mathrm{n}^{\circ}$ 1) es el desarrollado por Holbrook, MB. (1999) en donde se detallan ocho tipos de valor en tres dimensiones claves de consumo.

\begin{tabular}{|c|c|c|c|}
\hline \multicolumn{2}{|c|}{} & EXTRÍNSECO & INTRÍNSECO \\
\hline \multirow{2}{*}{ ORIENTADO HACIA SÍ MISMO } & ACTIV0 & $\begin{array}{c}\text { EFICIENCIA } \\
\text { (Conveniencia) }\end{array}$ & $\begin{array}{c}\text { ENTRETENIMIENTO } \\
\text { (Diversión) }\end{array}$ \\
\cline { 2 - 4 } & REACTIV0 & $\begin{array}{c}\text { EXCELENCIA } \\
\text { (Calidad) }\end{array}$ & $\begin{array}{c}\text { ESTÉTICA } \\
\text { (Belleza) }\end{array}$ \\
\hline \multirow{2}{*}{$\begin{array}{c}\text { ORIENTADO HACIA LOS } \\
\text { DEMÁS }\end{array}$} & ACTIV0 & $\begin{array}{c}\text { ESTATUS } \\
\text { (Éxito) }\end{array}$ & $\begin{array}{c}\text { ÉTICA } \\
\text { (Virtud, Justicia) }\end{array}$ \\
\cline { 2 - 4 } & REACTIVO & $\begin{array}{c}\text { ESTIMA } \\
\text { (Reputación, Materialismo) }\end{array}$ & $\begin{array}{c}\text { ESPIRITUALIDAD } \\
\text { (Fe) }\end{array}$ \\
\hline
\end{tabular}

Tabla 1: Tipología de Valor de Holbrook, M.B. (1999) citado por Gallarzar, M. y Gil, I. (2005).

Según Gallarza, M. y Gil, I. (2005) “la mayor cualidad de la tipología de Holbrook, M.B. (1999) es que en ella tienen cabida múltiples experiencias de consumo: afectivas, funcionales, intelectuales, físicas, mentales y otras," 


\section{MONOGRÁFICO}

Desde la perspectiva del Marketing es que Bonal, S. y Cuadrado, J. (2014) plantean que "el valor percibido por el cliente es una ecuación que pondera lo que el cliente obtiene de la marca y lo que le cuesta obtenerlo", por lo mismo los autores plantean una fórmula que permite describir los componentes del valor percibido y que a la vez sirven para identificar qué es lo que un cliente considera como valioso respeto de una marca y que se puede graficar de la siguiente manera:

Valor Percibido $=$ Prestaciones + Emociones

$$
\text { Precio + Incomodidades + inseguridades }
$$

De acuerdo a lo planteado por ambos autores se pueden comprender

a. Prestaciones: Todo lo que la marca hace por el cliente entre lo que se cuenta el producto o servicio central de la oferta y los servicios accesorios que complementan la oferta de valor. Se incluyen tanto elementos materiales (producto) como elementos inmateriales (servicio de atención técnica).

b. Emociones: Corresponde a las experiencias que busca el cliente quien quiere comprobar que lo que las marcas le comunican es verdad.

c. Precio: Es el importe de la contraprestación puramente económica que el cliente debe pagar para conseguir el producto o servicio.

d. Incomodidades: Es toda alteración o coste extra en el que el cliente debe incurrir y que afecta su relación con la marca

e. Inseguridades: Conjunto de percepciones que hacen que el consumidor se sienta inseguro en la relación con la marca.

Por todo lo anterior, es que se decidió que la ecuación planteada por Bonal S. y Cuadrado, J. (2004) se complementará con la Tipología de Holbrook, M.B. (1999) para abordar el valor percibido por los usuarios al momento de convertirse en seguidor de una marca en Facebook. 


\section{Material y Métodos}

Para realizar esta investigación se utilizó una metodología cuantitativa y el método de recolección de datos fue la aplicación de una encuesta auto administrada on line cuyo cuestionario fue validado por expertos en la materia y sometido a un pre test que aseguró la relevancia y claridad del mismo.

Siguiendo el mismo procedimiento utilizado en estudios sobre amor a la marca (Albert et al., 2009 y Alfonso et al., 2014) el muestreo se realizó a través del procedimiento bola de nieve, consistente en proporcionar un enlace web a una lista de contactos junto con un mensaje personal en el que se solicita su participación en el estudio y que difundan el link de la encuesta a sus propios contactos.

La recolección de datos se obtendrá mediante la aplicación de una encuesta autoadministrada on line cuyo cuestionario será validado por expertos en la materia y sometido a un pre test para asegurar la relevancia y claridad del mismo.

El cuestionario estará compuesto de 30 preguntas que exploran la frecuencia de uso de Redes Sociales, las motivaciones que tienen los jóvenes para seguir a una marca en Facebook y la valoración y utilidad percibida que tienen de las mismas. Para esto, se utilizaron preguntas de tipo dicotómicas y categóricas, así como también preguntas con grado de aceptación usando una escala de tipo Likert de cinco grados.

Los datos demográficos del cuestionario incluyen edad, sexo, Región en donde estudian y Tipo de Institución Superior en donde estudian.

\section{Resultados}

De los 1.117 encuestados en este estudio, 382 son hombres y 635 mujeres. Las edades fluctúan entre los 17 y los 29 años siendo la moda y la media 23 años.

En cuanto a los hombres, el $84,8 \%$ estudia en Universidades mientras que el 15,2\% lo hace en Centros de Formación Técnica o Institutos Profesionales. 


\section{MONOGRÁFICO}

Por su parte, en mujeres el $91,2 \%$ de la muestra estudia en Universidades y el 8,8\% lo hace Centros de Formación Técnica o Institutos Profesionales.

El $90 \%$ de la muestra sigue a marcas en sus redes sociales, el $8 \%$ no lo hace y el $2 \%$ no sabe o no responde a esta pregunta. Sobre este mismo punto, hay una leve diferencia al momento de dividir a la muestra por sexo, pues el $93 \%$ de las mujeres encuestas siguen a marcas en sus redes sociales y solo el $83 \%$ de los hombres lo hace. El $12 \%$ de los hombres encuestados declara no seguir a marcas, mientras que el $5 \%$ de las mujeres se comporta de la misma manera. Solo el 5\% de los hombres no sabe si sigue o no marcas en sus redes sociales mientras que en las mujeres este porcentaje es del $2 \%$.

El 94\% de los jóvenes usa, en promedio, tres redes sociales en su día a día y de éstas las más utilizadas son Facebook (39\%) Instagram (35\%) Twitter (20\%) Mucho más atrás en las preferencias aparece Snapchat $(3 \%)$ y prácticamente imperceptibles para los jóvenes están Pinterest (1\%) Tumblr (1\%) y WhatsApp (1\%)

Respecto de las tres redes sociales más utilizadas hay similitudes al momento de analizar por sexo. Facebook es preferida por el $44 \%$ de los hombres y por el $41 \%$ de las mujeres; Instagram por el 35\% de los hombres y el 38\% de las mujeres, mientras que Twitter se queda 21\% de ambos sexos.

Al momento de seguir a las marcas en redes sociales Instagram (47\%) y Facebook (46\%) son las preferidas por las mujeres. Twitter se queda con un $4 \%$ de las preferencias, mientras que el $3 \%$ no declara no tener una red social preferida para seguir a marcas.

En los hombres las redes sociales preferidas para seguir a marcas son Facebook (58\%) Instagram (25\%) Twitter (9\%). El 8\% restante no tiene una red social preferida.

En lo que se refiere a la credibilidad de las marcas en redes sociales, el $56 \%$ de los encuestados señala que le cree bastante, el 13\% mucho, el $27 \%$ poco y el $3 \%$ nada. El comportamiento por sexos es similar. En hombres, un $52 \%$ le cree bastan- 
te, un $14 \%$ mucho, un $28 \%$ poco y un $6 \%$ nada. En mujeres el $60 \%$ le cree bastante, el $12 \%$ mucho, el $26 \%$ poco y el $2 \%$ nada.

En lo referente a la publicidad de marcas en redes sociales el $65 \%$ de los encuestados señala estar a favor de que las marcas la realicen porque así se enteran de las novedades de sus productos, el 3\% considera que las redes sociales no son un espacio para realizar publicidad; mientras que el $32 \%$ se muestra indiferente ante la publicidad en redes sociales.

Para analizar el valor percibido se consideró la plataforma que usan para seguir a las marcas y el por qué la prefieren por sobre el resto. Así, se logró establecer una relación entre la plataforma y las posibilidades que éstas muestran para los usuarios al momento de hablar de valor percibido. En este análisis se consideraron las tres redes sociales que son más usadas por los jóvenes para seguir marcas: Facebook, Instagram y Twitter.

Las tres redes sociales presentan una orientación hacia sí mismos del tipo activa Facebook (70\%) Instagram (90\%) y Twitter (80\%) lo que significa que los jóvenes valoran el aspecto de consumo de manera egoísta, en este caso la plataforma, y las acciones que realizan en ella son las que configuran su experiencia de consumo. Esto revela que para los jóvenes el valor económico es el centro de la relación entre ellos y las marcas en redes sociales.

Lo más valorado por los encuestados, respecto de lo que realizan las marcas en redes sociales, es el contenido que comparten (64\%) que las marcas realicen promociones, concursos o den regalos (21\%) las respuestas y soluciones a problemas (8\%) y la utilidad de la información que publican (7\%)

Información (50\%) Regalos y Concursos (32\%) que los escuchen (12\%) que los acompañen (6\%) es lo esperan los jóvenes de las marcas que siguen en redes sociales. Esto según la tipología de Holbrook se enmarca dentro de una orientación así mismo del tipo activa en donde la conveniencia (eficiencia) es el principal factor motivador. 


\section{MONOGRÁFICO}

Las categorías cuyas marcas son más seguidas por los usuarios son Cultura y Medios de Comunicación (21\%) Estilos de vida (Deporte, salud, belleza, moda) (21\%) Telecomunicaciones (20\%) Comida y Diversión (13\%) y Tecnología (14\%) Bancos e Instituciones Financieras (5\%) 0tras (6\%)

\section{Discusión}

Considerando el estado de la literatura científica respecto de la relación entre las marcas y los usuarios a través de canales digitales aún está en desarrollo los resultados de este estudio aportan al conocimiento de los drivers que intervienen en el proceso de relación entre las marcas y los usuarios mediante canales digitales.

Los resultados de este estudio revelan que las motivaciones de los usuarios al momento de optar por seguir a una marca en redes sociales se basan principalmente en atributos hedonísticos (placeres) y esperan obtener recompensas de las marcas que se deben materializar en una propuesta de valor coherente con los valores y atributos de cada una de las marcas. Algo que está en relación a lo que plantea Alfonso et al (2015) que ahonda sobre el amor a las marcas y las representaciones de éstas.

Basado en lo que plantea de Notley, T. (2009) este estudio comprueba que en la realidad chilena no son aplicables las mismas escalas para medir la relación entre una marca y sus usuarios en canales digitales. Esto se puede explicar por el desarrollo de la industria digital en Chile es menor a la de Australia y que las características personales son diferentes por ser culturas distintas.

Siguiendo la línea de lo planteado por Zheng, R. y Cheok, A. (2011) los resultados de este estudio permiten tomar un punto de partida en las estrategias comunicacionales para las marcas apoyando los procesos de mejora continua de éstas lo que a la larga se puede traducir en mejoras para ellas.

Por último, este estudio incorpora nuevos resultados y complementa al realizado por Soengas, X., Vivar, H. y Abuín, N (2015) respecto de la relación entre marcas y usuarios en canales digitales. 
Desde el punto de vista de la comunicación estratégica y considerando que el estudio "Inmersion" elaborado por la Agencia Digital Jelly y CADEM (2015) reveló que el $90 \%$ de las empresas chilenas tienen una estrategia de marketing digital y que en promedio los ejecutivos ponen una nota de 5,7 (en escala de 1 a 7 ) a la importancia del marketing digital y las redes sociales al momento de planificar la estrategia de marca se sugiere que:

1. Es útil entender que los usuarios esperan una mantener una relación con las marcas en redes sociales y que por lo mismo las marcas deben interactuar como un usuario más en el canal digital que escojan y que para esto el contenido que compartan es fundamental. El 50\% de los encuestados considera que el contenido es uno de los aspectos fundamentales de las marcas en redes sociales.

2. Es necesario mejorar la credibilidad de las marcas. Considerando que el $30 \%$ de los usuarios que cree poco o nada a la comunicación que realizan las marcas en canales digitales parece interesante reforzar este atributo mediante acciones especiales que le permitan al usuario reconocer a las marcas en este nuevo entorno.

3. Es necesario capitalizar las acciones realizadas. Considerando que el $65 \%$ de los encuestados está de acuerdo en que las marcas realicen publicidad en redes sociales y que el $32 \%$ valora que las marcas realicen concursos, premios y regalos en canales digitales, parece importante establecer una relación que permita aumentar el ROI en cada una de las acciones realizadas, así como también establecer métricas que permitan aumentar el costumer value a través del RFM y los procesos de fidelización de los usuarios.

Entonces, considerando la realidad actual de las empresas chilenas que utilizan medios digitales para desarrollar su comunicación y que además contemplan en su público objetivo mayoritariamente a jóvenes, es posible señalar que las estrategias comunicacionales de éstas deben ser revisadas para generar un perfil más centrado a los desafíos actuales de las marcas considerando las valoraciones y las percepciones que tienen los usuarios de los modelos actuales. 


\section{MONOGRÁFICO}

Lo anterior, lleva a pensar que las marcas chilenas en redes sociales están realizando esfuerzos que son tan valorados como ellos creen por parte de los jóvenes. Esto, se ejemplifica en el tránsito que han realizado, prácticamente todas las marcas independiente de la categoría, al uso de canales digitales solo como contact center, dejando de lado la generación de comunidades en torno a ellas, de las cuales pudieran extraer insights de valor para la generación de sus estrategias.

Desde ese punto de vista, es necesario establecer cuál es el verdadero rol que juegan los usuarios digitales en la relación marca-cliente y qué peso específico tienen al momento del diseño e interacción de las marcas con sus clientes finales. A la luz de los resultados de este estudio, es posible señalar que las marcas no están dándole el valor requerido a sus usuarios, descuidando a quienes es necesario mantener y atiendo a quienes no presentan oportunidades claras de crecimiento. Esto, se basa en el análisis de los concursos y premios que realizan las marcas en redes sociales, que son monopolizados -en la mayoría de los escenarios- por usuarios de poco valor estratégico y real para las marcas quienes de cierta manera hacen poco visibles a quienes si tienen un valor estratégico o real para las marcas más alto, pero el número de interacciones (repeticiones de éstas) y el número de usuarios que los siguen (popularidad) muchas veces juegan un papel relevante aun cuando no sean clientes o parte del target de la marca y solo quieran la recompensa que se está entregando.

Es justo, entonces, plantearse, cómo las marcas diseñan y modelan las interacciones que desean tener con sus usuarios y cómo de éstos pueden obtener retroalimentaciones que les permitan cumplir objetivos comerciales y no solo hablar de prioridades como alcance y visibilidad sin priorizar otros elementos comunicacionales que pudieran tener más valor para la marca.

\section{Limitaciones y líneas futuras que se derivan del trabajo}

Como toda investigación, en esta también hay algunas limitaciones que dejan la puerta abierta al desarrollo de futuros trabajos que traten ciertos aspectos que a continuación se detallan. 
En primer lugar, hay que señalar que dado el tamaño de la muestra, los resultados obtenidos no pueden ser generalizados. Sería conveniente, replicar el estudio con una muestra más grande para ofrecer garantías sobre la validez externa del estudio, considerando factores geográficos al momento de la selección. Algo similar ocurre con el sexo de los sujetos.

En segundo lugar, y considerando que la relación de la marca con los usuarios a través de canales digitales es un factor determinante para la industria en general se plantea como óptimo incluir ciertas variables que permitan medir el Top of Mind (T.o.M) de los usuarios y no sólo establecer las categorías de las marcas.

Finalmente, y considerando el escaso número de estudios en esta materia desarrollados hasta el momento, resulta interesante fijarse en temáticas no abordadas, como por ejemplo ¿Cuánto influyen los grupos de referencia al momento de establecer una relación con una marca en Redes Sociales? ¿Qué papel juegan las Redes Sociales en la generación de imaginarios colectivos para las marcas? ¿Puede una correcta comunicación a través de Redes Sociales influir en el ciclo de compra de un consumidor? ¿Qué tipo de características individuales tienen las personas que siguen a una marca en Redes Sociales?

\section{Referencias}

Anderson, J.C. (1995). “Relationships in Business Markets: exchange episodes, value creation, and their empirical assessment". Journal of the Academy of Marketing Science, Vol. 23, No 4, pp. 346-350.

Babin, B.J., Darden, W.R. y Griffin, M. (1994). “Work and/or fun: measuring hedonic and utilitarian shopping value". Journal of Consumer Research, Vol. 20 (March), pp. 644-656.

Barford, I. N., \& Hester, P. T. (2011). Analysis of Generation Y workforce motivation using multiattribute utility theory. Defense Acquisition Research Journal.

Bernal, C. y Angulo, F (2012) Interacciones de los jóvenes andaluces en las redes sociales. Revista Científica de Educomunicación. Volumen XX

Berry, L.D. y Yadav, M.S. (1997). “El papel del valor en la determinación del precio de los servicios". Harvard Deusto Business Review, No 78, pp. 26-37. 


\section{MONOGRÁFICO}

Bigné, J.E., Moliner, M.A. y Callarisa, L.J. (2000). “El valor y la fidelización de clientes: propuesta de modelo dinámico de comportamiento". Revista Europea de Dirección y Economía de la Empresa, Vol. 9, № 3, pp. 65-78.

Bonal, S. y Cuadrado, J. (2014) ¿Cuál es la percepción del valor para el cliente? Disponible en http://blogs.icemd.com/blog-customer-centric-marketing/ cual-es-la-percepcion-de-valor-del-cliente/ Consultado en diciembre de 2015.

Butz, H.E. y Goldstein, L.B. (1996). "Measuring customer value: gaining the strategic advantage". Organizational Dynamics, Vol. 24 (Invierno), pp. 63-77, cit. en DAY y CRASK (2000).

Carvallo, P (2014) Estudio de los millenials chilenos en el mercado laboral. Tesis de grado para optar al grado de Magíster en Gestión de Personas y Dinámica Organizacional. Posgrados Facultad de Economía y Negocios. Universidad de Chile. Castelló, A. (2010) ¿Cuánto vale un fan? El reto de la medición de la audiencia en los social media. Pensar la Publicidad. Volumen IV 89-110

Colás, P. González, T. y de Pablos, J. (2012) Juventud y redes sociales. Comunicar, $\mathrm{n}^{0}$ 40, v. XX, 2013, Revista Científica de Educomunicación; ISSN: 1134-3478; páginas 15-23. comScore Inc. (2015) Futuro Digital Foco en Chile.

Connell, J., McMinn, N. E., \& Bell, N. (2012). How will the next generation change de business world? A report on a survey. Insights to a Changing World Journal.

Cruz, B. y Mendelsohn, J. (2010) Why Social Media Matters Matters to Your Business. Disponible en http://www.cmbinfo.com/cmb-cms/wp -content/ uploads/2010/04/Why_Social_Media_Matters_2010.pdf Consultado en diciembre de 2015.

Dodds, W., Monroe, K. y Grewal, D. (1991). "Effects of price, brand, and store information on buyers' product evaluations". Journal of Marketing Research, Vol. 28, pp. 307-19.

Gabbott, M. y Hogg, G. (1998). Consumer and Services. Ed. Wiley, Chichester.

Gallarzar, M. y Gil, I. (2005) Desarrollo de una escala multidimensional para medir el valor percibido de una experiencia de servicio. Revista Española de Investigación de Marketing ESIC pp 25 - 59

Gil, I; Sanchez, M; Berenguer, G; Gonzalez-Gallarza, M (2006) "Encuentro de servicio, valor percibido y satisfacción del cliente en la relación entre empresas", Cuadernos de Estudios Empresariales 15, 47-72 
¿Qué esperan obtener los jóvenes chilenos de las marcas que siguen... | 137

MONOGRÁFICO

Holbrook, M.B. (1999). Consumer value. A framework for analysis and research. Ed. Routledge, Londres.

IAB Spain Research (2015) Estudio sobre Redes Sociales en Internet. Disponible en http://es.slideshare.net/elogia/estudio-redes-sociales-iab-spain-2015 Consultado en diciembre de 2015.

Igarza, R. (2008) Nuevos medios: estrategia de Convergencia. La Crujía Ediciones.

Lehr, A. (2015) The Unfollow Algorithm. Disponible en http://www.buzzstream. com/blog/unfollow-algorithm.html Consultado en diciembre de 2015.

Martínez, E. y Sánchez, L. (2011) Publicidad en Internet: Nuevas vinculaciones en las redes sociales. Revista de Comunicación Vivat Academia. Volumne 117E pp 469-480

Monroe K. B. (1992) Política de precios. Para hacer más rentables las decisiones, McGraw-Hill Management, Madrid.

Muela, C. y Baladrón, J. (2010) Jóvenes y Publicidad on line: nuevos espacios y formas, otros retos. Documentos Área de Publicidad Facultad de Ciencias de la Comunicación (URJC) pp 183-199

Nilson, T.H. (1992) Value-added marketing: marketing management for superior results McGraw Hill. Berkshire. UK.

Notley, T. (2009) Young People, Online Networks, and Social Inclusion. Journal of Computer-Mediated Communication, 14, pp. 1208-1227.

Paz, C. Vázquez, R., Santos, L. (2010) Publicidad y Eficacia Publicitaria: influencia de la posición, repetición y estilos publicitarios en la eficacia de los anuncios televisivos entre los jóvenes. Universidad de Oviedo. Departamento de Administración de Empresas y Contabilidad.

Rogers, M. (2014) 5 razones que motivan el uso de redes sociales en Latinoamérica. Disponible en http://www.merca20.com/5-razones-que-motivan-el-uso-deredes-sociales-en-latinoamerica/ Consultado en diciembre de 2015.

Rosales, P. (2010) Estrategia Digital, cómo usar las nuevas tecnologías mejor que la competencia. Editorial Deusto 